\title{
"Jacques le fataliste" Diderot et le roman
}

\section{Lucie Bryant}

Le dix-huitième siècle est une période de transition. Les genres dramatiques se modifient ou se meurent. Le roman se forme par tâtonnements. I1 tend d'abord vers 1 'histoire et vers les mémoires puis s'oriente vers des genres nouveaux comme le conte philosophique et le conte moral. Voilà donc une richesse de problèmes offerts à la réflexion des romanciers et particulièrement à la réflexion de Diderot.

Nous allons étudier comment Diderot envisageait $1 e$ beau et la création romanesque et pour quelles raisons cette création devait différer de celle de ses prédécesseurs. Quelles devaient être selon lui les qualités et même peut-être les défauts d'un romancier? Nous allons tenter d'évaluer dans quelle mesure il a appliqué ses théories à la création d'un de ses romans, écrit en 1773 et publié chez 1'éditeur Buisson en 1796: Jacques le fataliste.

Dès le début de son oeuvre, Diderot nous avertit que Jacques le fataliste n'est pas un roman, du moins pas un roman tel qu'on en composait avant lui.

"Il est bien évident que je ne fais pas un roman puisque je néglige ce qu'un romancier ne manquerait pas d'employer. Celui qui prendrait ce que j'écris pour la vérité serait peut-être moins dans $1^{\prime}$ erreur que celui qui le prendrait pour une fable." (p. 43)

Voilà le mot important pour Diderot: faire vrai. La nature nous offre mille modèles où choisir notre matière.

"La nature est si variée surtout dans les instincts et dans les caractères qu'il n'y a rien de si bizarre 


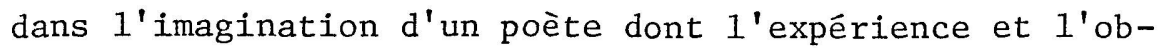
servation ne nous offrissent de modèle dans la nature." (p. 90)

Il faut faire simple, grand et large par la varié,té qui frappe, la masse qui impose. Puisée dans la nature, cette masse engendre des idées de durée, de puissance et de solidité. Seule l'expression immédiate et directe des passions dans leur forme spontanée lui semble être vraie et naturelle. C'est que pour lui l'art doit représenter la vie dans tout son flux et pour lui, la vie ce sont $1^{\prime}$ amour et la nature grâce auxquels il peut échapper à une civilisation artfficielle et brutale, génératrice de misère et de mort. Après la contrainte du XVII e siècle, Diderot veut libérer l'homme au nom de l'instinct et de la science.

La nature est $1^{\prime}$ ensemble des lois qui nous régissent et elle est matière d'art. Il faut la décrire, mais les descriptions doivent être subordonnées à un sentiment qui inspirera un personnage et elles doivent surtout représenter une richesse d'impressions. La nature instruit 1 'homme et lui suggère des idées philosophiques en multipliant ses sensations, surtout s'il s'agit d'un homme de génie. L'enthousiasme est la condition même de I'oeuvre d'art. L'inspiration naît de la passion, elle se traduit par un trouble psychique qui jette 1 'homme de génie hors de lui-même et lui fait saisir la complexité de 1 'homme et de son destin.

Pendant toute sa vie, Diderot a été conscient des nombreux paradoxes qui empêchent 1 'homme de génie de parvenir à une vérité définitive. Un des grands problèmes qui a préoccupé Diderot est celui de la liberté, liberté pour 1 'homme d'atteindre au bonheur qui est surtout fait de la connaissance de son être intime mais aussi de ses relations en tant qu'homme avec le monde extérieur. Dans Jacques le fataliste, Diderot présente les deux aspects de la question de la liberté. Le mầtre de Jacques se sent libre intuitivement et il croit à la liberté. Jacques, lui affirme que c'est une illusion et que "tout est marqué sur le grand rouleau." Tout au long du roman Jacques montre 1 'enchaînement d'une série d'épisodes qui sont en apparence commandés par un destin implacable et aveugle. Cependant notre sympathie va au maître qui veut reconnaître à chaque 
homme une essence personnelle, unique, capable de s'améliorer par 1'effort indépendant du coeur et de $1 \mathrm{a}$ volonté.

Ayant présenté ces deux positions opposées, Diderot laisse entendre que Jacques et son maître ont tort tous les deux. Il est ridicule de vivre comme si tout était inécaniquement organisé mais croire à notre liberté est une illusion. Il y a là un conflit que Diderot n'a jamais réussi à résoudre et il pense que l'homme ne peut trouver de solution définitive, du moins dans cette vie terrestre.

"Qui sait cela avant que d'être arrivé au dernier mot de la dernière ligne de la page qu'on remplit dans le grand rouleau?" (p. 287)

La dignité de 1 'homme est donc pour Diderot dans la recherche et non dans la découverte de la vérité. L'unité de 1'homme et surtout de 1'écrivain peut se trouver dans le combat qu'il mène pour rechercher et présenter la vérité relative dans toute sa multiplicité. Ainsi compris le destin de l'homme s'exprime en paradoxes qui permettent à Diderot de proposer à ses lecteurs les aspects divers d'une même question et aux lecteurs d'avoir une plus grande connaissance des problèmes traités. Un paradoxe trouble aussi le lecteur en lui proposant un choix et le force à réfléchir. Diderot oblige le lecteur à participer activement à la recherche de la vérité au lieu de suivre l'opinion arrêtée des auteurs du passé.

"Un paradoxe n'est pas toujours une fausseté. Soyez circonspect si vous ne voulez pas prendre dans cet entretien de Jacques et de son mâ̂tre le vrai pour le faux et le faux pour le vrai." (p. 90)

Voilà justement une des grandes difficultés pour l'écrivain qui veut faire vrai; la difficulté de définir la vérité. Peut-être un événement était-il vrai ou peut-être faux? sait-on jamais? Un écrivain selon Diderot ne doit pas être un fade panégyriste ni un censeur amer, mais doit offrir les faits comme ils sont et 1a chose n'est pas aisée.

"Mon cher maitre, la vie se passe en quiproquos. I1 y a les quiproquos d'amour, d'amitié, les quiproquos politiques, de finance, de magistrature, d'église, de commerce, de femmes et de maris..." (p. 81) 
Diderot accuse le monde de ne pas comprendre non seulement les intentions mais aussi les actions, qui, elles, devraient être faciles à évaluer.

"Si 1 'on ne dit presque rien dans le monde qui ne soit entendu comme on le dit, il y a bien pis, c'est qu'on n'y fait presque rien qui soit jugé comme on $I^{\prime}$ a fait." (p. 81)

Un autre aspect du problème de la difficulté de saisir la vérité èt du problème de la liberté est traité avec insistance par Diderot. Le maître croit à la possibilité d'un acte libre, choisi sans motif, sans intérêt personnel vital, un acte gratuit, accompli seulement pour se prouver qu'on est libre. Diderot montre qu'un tel acte est une illusion car un acte n'est jamais isolé, il est toujours suivi de conséquences que l'auteur de I'acte ne peut prévoir. Ainsi le mâ̂tre a raconté à Jacques les conséquences inattendues de la nuit qu'il avait choisie de passer avec Agathe. Diderot conclut alors:

"On passe les trois-quarts de sa vie à faire sans vouloir et à vouloir sans faire." ( p. 288) Diderot insiste plusieurs fois sur l'impuissance de 1 'homme à choisir son destin. "Faute de savoir ce qui est écrit là-haut, on ne sait ni ce qu'on veut ni ce qu'on fait et on suit sa fantaisie qu'on appelle raison ou sa raison qui n'est souvent qu'une dangereuse fantaisie qui tourne tantôt bien tantôt mal." ( p. 40)

I1 faut se rendre compte, nous dit Diderot, que si 1 'on pose une cause, un effet s'ensuivra et que d'une cause faible naît un effet faible, d'une cause momentanée, un effet momentané et cela souvent sans notre accord. Un fou seul, dit Diderot peut user de sa liberté sans motif.

Diderot veut que 1'artiste écrive pour être utile, pour enseigner aux hommes la situation paradoxale dans laquelle ils se trouvent, pour aider au progrès intellectuel, social et moral. Il ignore le dilettantisme et l'art pour l'art. Il brûle de communiquer ses impressions. Instruire est pour lui civiliser mais il faut avant tout attirer $1^{\prime}$ attention du lecteur sur 1a diversité de la vérité. Il est nécessaire également de plaire, d'être agréable. 
"La vérité est souvent froide, commune et plate. Un récit peut être vrai, mais pas intéressant. S'il faut être vrai c'est comme Molière, Regnard, Richardson, Sedaine. La vérité a des côtés piquants qu'on saisit quand on a du génie." (p. 64)

Diderot veut plaire et amuser pour faire réfléchir par des réflexions contradictoires. Il dit qu'il est facile de faire des contes car le conteur a la liberté de jouer avec ses personnages. Il est un dictateur qui tire les êtres du néant, les dirige suivant un ordre établi et des fins fixées à l'avance. Rien n'est, dans les contes classiques, laissé au hasard, tout est justifié mais Diderot veut renoncer aux préjugés, à la tradition, à 1'autorité. I1 rejette tout ce qui rend 1 'esprit prisonnier. Il veut avoir 1'audace de penser par lui-même. Il ne veut pas prononcer de jugements définitifs. Il veut présenter des rapports entre diverses idées. Dans L'Essai sur le mérite et la vertu, Diderot avait déjà exprimé cette idée de rapports en définissant sa conception du beau:

"J'appelle beau hors de moi, tout ce qui contient en soi de quoi réveiller de mon entendement l'idée de rapports et beau par rapport à moi, tout ce qui réveille cette idée."

Diderot utilise son intelligence pour établir des rapports et percevoir des analogies. Il aime tirer d'un principe ses conséquences les plus opposées et les plus extrêmes. Ses connaissances scientifiques lui ont révélé une foule de formes vivantes passant de l'une à l'autre dans un ordre croissant de complexités et de nouvelles espèces découvertes chaque jour, d'où la nécessité d'assouplir les ordres de classification. Diderot en tire la conséquence qu'en littérature de même les règles $n$ 'ont aucun caractère sacré. Elles consacrent les coutumes des siècles passés mais il faut créer de nouveaux types.

Des sciences naturelles Diderot a retenu qu'aucune interprétation ne résiste aux résultats d'une expérience. La loi, née en laboratoire n'a d'autre utilité que de permettre de nouveaux essais. L'expérience propre, l'intérêt présent et la voix de la conscience voilà les grands professeurs de la vie. Diderot a substitué à la notion abstraite de 1 'homme en général, la réalité vi- 
vante de l'être de chair, de ses faiblesses, de ses contradictions mais aussi de son inlassable curiosité.

Se refusant à ériger ses idées en système puisque la vérité est toujours variable et sans cesse à poursuivre, Diderot demande au romancier d'être un homme de goût. Ce goût est le fruit de l'expérience, d'une infinité d'expériences qui se réveillent devant un objet nouveau et déterminent pour un temps bref le jugement qu'on leur porte. I1 faut rechercher la vérité sans cesse. Pour cela 1'artiste doit par la réflexion et l'étude, mêlant l'expérience à la méditation, se libérer des préjugés de son éducation et de la société qui l'entoure. Il doit s'efforcer de se créer un univers intérieur où 1 'unité et 1 'harmonie sont créées par le désir d'être ouvert à toutes les possibilités humaines. La grande règle est la convenance c'est à dire l'adaptation exacte de 1 'ouvrage à son objet. Dans Jacques le fataliste, Diderot montre que la vérité est complexe diverse, contradictoire. I1 rend compte des aspects opposés de chaque question, ici du problème de la liberté, et sa curiosité, son esprit toujours en mouvement, sondent les diverses possibilités qui se présentent à la liberté humaine, mais aussi ses limitations. Il s'intéresse plus au problème qu'à la solution, nous avons vu plus haut qu'il ne croit pas aux solutions définitives. C'est pourquoi l'examen des opinions diverses sur un même sujet s'offre à lui sous la forme d'un dialogue, d'une discussion. On sent dans son effort de persuader le besoin de se persuader lui-même et d'aller au-delà des apparences. Le crime contre $1^{\prime}$ esprit pour Diderot c'est une défaillance dans la recherche de la vérité.

Dans Jacques le fataliste, Diderot divise son moi entre divers interlocuteurs. Jacques incarne son goût de $1^{\prime}$ aventure intellectuelle, sa satire du déterminisme, son admiration pour I'instinct sexuel qui est nécessairement bon, et doit être libre. Jacques et son mâttre critiquent la civilisation millénaire qui gâte I'homme et lui apporte une déchéance physique et morale. Diderot assiste au débat, partie et juge. Il contemple ses propres transports et ni sa spontanéité ni sa clairvoyance ne sont altérées.

La recette de Diderot pour créer ce qui n'est pas 
un roman traditionnel mais un roman philosophique apparaît assez simple. Il se sert de supports romanesques plaisants ou, si possible, frappants pour offrir une thèse philosophique ou une critique des moeurs, par exemple dans Jacques le fataliste. Il attaque tout ce que la société de son temps avait appris à respecter. Sa critique est aigüe, lucide, dirigée par une impétuosité sans frein. Il doit en effet se débarrasser des préjugés anciens avant de continuer sa recherche de la vérité. Ce récit endiablé contient une leçon morale, sociale ou philosophique qu'il appartient au lecteur de dégager.

"Quelque soit le récit que tu m'as promis, sois sûr qu'il ne sera vide d'instruction que pour un sot." (p. 233)

Diderot a critiqué la littérature post-classique où tout, disait-il est ordonné, conventionnel et par conséquent plat et ennuyeux. Lui-même a tenté 1'inverse en emplissant son oeuvre d'aventures piquantes et apparemment désordonnées et même, disons le mot, d'obscénités dont d'ailleurs il s'est défendu en disant qu'il voulait faire vrai et que la nature ne faisait rien d'inutile ni de superflu. Un écrivain doit avoir le courage de présenter les choses comme elles sont.

Toujours pour être conforme à cette vérité vivante, Diderot n'a pas rempli son récit des retours préparés avec soin des divers personnages. Il ne veut pas faire revenir sur la scène de son roman un personnage pour l'unique raison de continuer son récit avec harmonie. Souvent ses personnages apparaissent, représentent une idée et leurs actions sont choisies dans ce but, puis ils disparaissent pour toujours du roman et d'autres personnages font leur apparition pour quelques pages. Tout doit rester vrai et la vie est complexe. Pour cette raison il hait les portraits qui ne ressemblent pas aux originaux.

"Racontez les faits, rendez-moi fidèlement les propos et je saurai bientôt à quel homme j'ai à faire. Un mot, un geste m'ont parfois plus appris que le bavardage de toute une ville." ( $p$. 282) Pour la même raison il n'aime pas non plus les descriptions. "Ah. Jacques fais-moi grâce, je t'en prie et de la description de la maison et du caractère du docteur et de 1'humeur de la 
doctoresse et des progrès de ta guèrison; saute, saute par-dessus tout cela." (p. 105)

I1 faut présenter les choses sans rien omettre ni rien ajouter et il refuse dans Jacques le fataliste d'insérer 1a lettre d'Agathe, 1'amie du mâ̂tre de Jacques, lettre qui, dit-il se lit avec plaisir mais détruit l'idée présentée par le roman.

Pour Diderot, un roman traditionnel est une histoire dont le fond est inventé et dont la forme est arrangée, par conséquent une histoire doublement fausse. Diderot, lui, écrit une histoire doublement vraie, car il ne dit que ce qui arrive (voilà pour le fond) et à mesure que cela arrive, (voilà pour la forme). En raison de sa devise: "Vérité, vérité." Diderot se doit de présenter une histoire décousue, faite de mille événements hautement colorés qui s'échappent sans cesse et se rejoignent souvent on ne sait comment. A tout moment 1'auteur est interrompu, s'interrompt lui-même et interrompt ses interruptions. I1 y a des coqs-à-I'âne saugrenus. Diderot explique que c'est pour mieux imiter la nature qui ignore $1^{\prime}$ art des transitions et ne s'efforce pas de bien comparer et de bien organiser. On a reproché à Diderot son manque d'ordre, son style négligé, et 1 'absence de composition de son oeuvre, mais il a pratiqué le désordre de façon consciente et même systématique et quand son tempérament enthousiaste l'emportait c'était un atout de plus pour son art de romancier.

Il y a une qualité nécessaire à l'écrivain, que Diderot souligne, c'est la sensibilité. La sensibilité confère à $1^{\prime}$ homme sa noblesse d'âme et $1^{\prime}$ excite à $1^{\prime}$ 'action et à $I^{\prime}$ enthousiasme mais Diderot pense que la sensibilité est également dangereuse car une trop grande sensibilité obscurcit parfois les notions de vrai et pousse un écrivain à l'erreur; cependant un homme ne peut être génial sans une âme sensible.

Diderot a produit une oeuvre originale qui a su envisager les problèmes du roman au dix-huitième siècle. Il n'a pas résolu les questions qu'il s'est posées, il n'a pas pu établir une esthétique absolue mais il a cherché à nous présenter les données de certains problèmes esthétiques. Il a montré que pour connaître un homme il fallait être arrivé au dernier mot que l'on 
écrit sur le grand rouleau et que la vie est mouvante, fluide sans cesse renouvelée. Par les nombreuses digressions, les nombreuses aventures de Jacques le fataliste il a offert différentes facettes de 1'homme, il a aussi proposé diverses solutions mais il a affirmé qu'il pouvait y avoir d'autres solutions aussi valables.

"Jacques a dit cent fois qu'il était écrit là-haut qu'il n'en finirait pas de l'histoire. Je vois, lecteur, que cela vous fâche; eh bien, reprenez son récit où il l'a laissé et continuez-le à votre fantaisie." (p. 305)

Diderot échappait avec Jacques le fataliste aux cadres rigides d'une classification, ici raison, ici sentiment. Il devait suivre le devenir des âmes et des esprits et montrait la coexistence de deux forces qui allaient devenir à nouveau divergentes. Le dix-neuvième siècle allait tourner le dos aux théories romanesques de Diderot mais à la veille de la première guerre mondiale, André Gide devait libérer 1'art du roman selon des lignes de pensées analogues.

\section{Bibliographie}

Diderot, Denis. Jacques le fataliste (Collection Dilecta, Editions Albin Miche1, Paris, 1963). Toutes les références à Jacques le fataliste se rapportent à cette édition et seront désormais indiquées dans le texte.

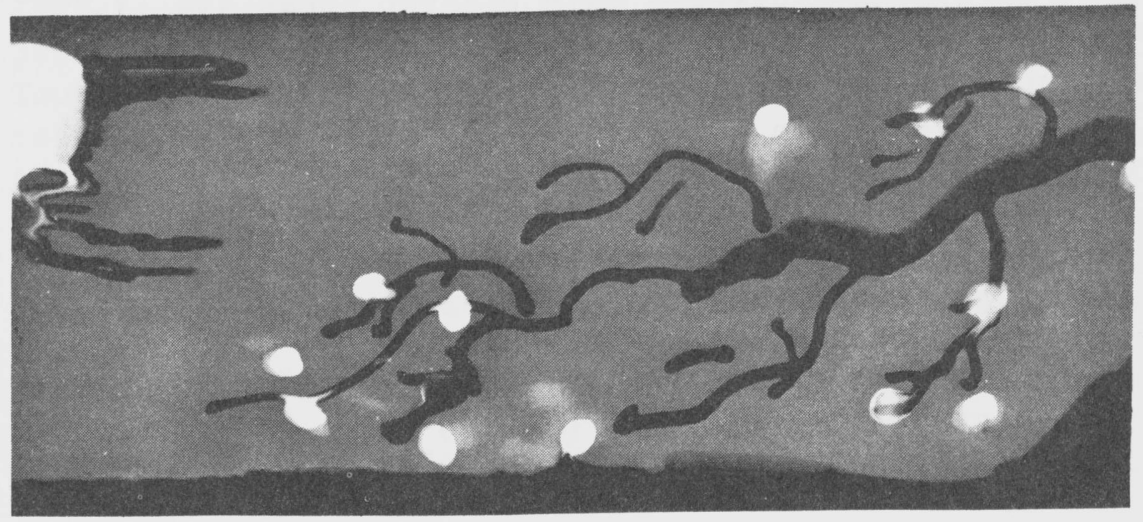


Votre tram est un paysage choisi

òu s'encaquent des mégères fantasques

Jouant du coude, sapant le bibi,

Sinistres sous leurs accoutrements flasques.

Tout en se plaignant "̀ chaque rumeur

L'estomac creux, 1a faim inopportune

Pourpres, dégoulinantes de sueur

Leurs bélements se mêlent au clair de lune.

Au clair de lune électrique et beau

Frénétiquement, non loin des arbres

Elles s'agitent comme des jets d'eau

Et ne ressemblent en rien à des marbres. 